JOURNAL OF

SYMPLECTIC GEOMETRY

Volume 1 Number 4, 829-850, 2002

\title{
EXISTENCE OF PERFECT MORSE FUNCTIONS ON SPACES WITH SEMI-FREE CIRCLE ACTION.
}

\section{MikHail KOGAN}

Let $M$ be a compact oriented simply-connected manifold of dimension at least 8. Assume $M$ is equipped with a torsion-free semi-free circle action with isolated fixed points. We prove $M$ has a perfect invariant MorseSmale function. The major ingredient in the proof is a new cancellation theorem for invariant Morse theory.

\section{Introduction.}

It is well known that the classical results of Morse theory $[\mathbf{8}, \mathbf{9}]$ do not directly generalize to the invariant case. In particular, a compact manifold with a compact group action does not necessarily have an invariant Morse-Smale function. (We say that an invariant function is an invariant Morse function if every connected component of the set of critical points is a nondegenerate group orbit and we call it Morse-Bott if every connected components of the set of critical points is nondegenerate, but does not necessarily contain only one group orbit. An invariant Morse (or Morse-Bott) function is Morse-Smale (respectively Morse-Bott-Smale) if the stable and unstable manifolds intersect transversely.) As a result, in the presence of a group action there is no guarantee of existence of perfect invariant Morse functions for which the invariant Morse inequalities become equalities making the invari-

ant Morse polynomial of the function equal to the invariant Poincare polynomial of the manifold. 
At the same time, there are plenty of examples, many of which appear in symplectic geometry, of manifolds with groups actions which carry nonconstant perfect invariant Morse-Bott functions. As shown by Atiyah and Bott $[\mathbf{1}, \mathbf{2}]$ all compact symplectic manifolds with Hamiltonian torus action are equipped with perfect invariant Morse-Bott functions which arise as components of moment maps.

The existence of perfect Morse-Bott functions is one of the many topological properties of Hamiltonian spaces for which it is natural to ask about a generalization to non-Hamiltonian actions. Some of these properties, the Kirwan surjectivity theorem [6] and the JeffreyKirwan localization formula [5], have been studied by the author [7] for non-Hamiltonian actions. It was shown that the Jeffrey-Kirwan localization formula can be extended to the case of equivariantly formal torus actions, while the Kirwan surjectivity theorem does not generalize to this case.

This paper, an attempt to generalize the existence of perfect invariant Morse-Bott functions to non-Hamiltonian actions, was motivated by the recent results of Hattori [4] and Tolman-Weitsman [10] on semi-free circle actions on symplectic manifolds. Tolman and Weitsman showed that every semi-free symplectic circle action on a compact symplectic manifold with finite nonempty fixed point set must be Hamiltonian and hence has a moment map, a perfect invariant Morse function.

Our main theorem generalizes this result to spaces with semi-free action whose equivariant cohomology is $\mathbb{Z}$-torsion-free. For the definitions of all the terms used in the statement of the theorem see Section 2.

Theorem 1.1. Let $M$ be a compact oriented simply-connected manifold with torsion-free semi-free circle action which has finitely many fixed points. Assume $\operatorname{dim} M \geq 8$. Then there exists a perfect invariant Morse-Smale function on $M$.

While the original motivation for this result comes from symplectic geometry, the methods of proof are borrowed from classical Morse theory, namely the ideas used in the proof of the $h$-cobordism theorem in [8]. The major ingredient in the proof is a new invariant cancellation theorem (Theorem 2.2) which resembles the cancellation theorem in Morse theory. The same way the cancellation theorem in Morse theory supplies the tools for eliminating extra critical points of Morse 
functions, the invariant cancellation theorem helps to eliminate extra critical circles of invariant Morse functions.

The essential idea behind this cancellation theorem is illustrated in Figure 1, where a critical circle of index 1 is cancelled by increasing the index of a critical point from 0 to 2. The action of the circle on both pictures in Figure 1 is given by a rotation around the vertical axis, while the Morse function is given by projection onto the same axis. In the first picture there are a critical point $p$ of index 0 and a critical circle $s$ of index 1 , while in the second picture $s$ is eliminated by increasing the index of $p$ to 2 .
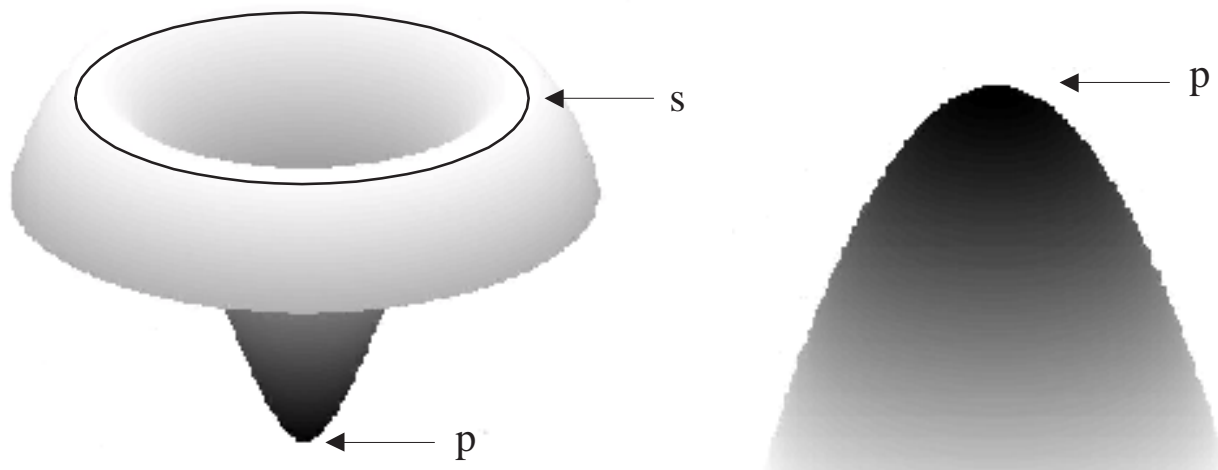

Figure 1: Critical circle $s$ is cancelled by increasing the index of critical point $p$ from 0 to 2 .

The paper is organized as follows. In Section 2 we provide basic definitions and notations, and state the main results of the paper. Section 3 contains the proof of existence of Morse-Smale functions on spaces with semi-free circle actions (Theorem 2.1). Section 4 provides the proof of the cancellation theorem (Theorem 2.2). The connections between invariant Morse theory and equivariant cohomology are discussed in Section 5. Finally, the last section is concerned with the proof of Theorem 1.1, which boils down to eliminating critical circles using the cancellation theorem. 
A word of warning to our readers: Since Morse theory is a vast subject, we have not cited all relevant sources for the results we will use below. For the most part we've quoted these results as described in the book of Milnor [8]. Also to shorten the presentation we have not included all details of the proofs of the equivariant analogues of standard Morse theory results but have simply indicated in outline how to adapt these results, as described in [8], to the equivariant case.

\section{Definitions and Main Results.}

A smooth action of a circle $S^{1}$ on a manifold $M$ is called semi-free if the action is free outside the set of fixed points $M^{S^{1}}$. In this paper we always assume that the action of $S^{1}$ on $M$ is semi-free and there are finitely many fixed points of the action.

An important property of semi-free actions is that around every isolated fixed point $p$, there exists an open neighborhood $U$ of $p$ and an invariant diffeomorphism $\phi$ between $U$ and an invariant open subset of $\mathbb{C}^{n}$, where $S^{1}$ acts on $\mathbb{C}^{n}$ with weight $(1, \ldots, 1)$. We call $(U, \phi)$ a standard chart at $p$. We will use both complex coordinates $\left(z_{1}, \ldots, z_{n}\right)$ and real coordinates $\left(x_{1}, y_{1}, \ldots, x_{n}, y_{n}\right)$ on $\mathbb{C}^{n}=\mathbb{R}^{2 n}$ with $z_{i}=x_{i}+\sqrt{-1} y_{i}$.

Let $f: M^{2 n} \rightarrow \mathbb{R}$ be a smooth invariant function. Denote by $\operatorname{Crit}(f)$ the set of critical points of $f$. Let $\operatorname{Crit}_{c}(f)$ be the set of connected components of $\operatorname{Crit}(f)$. Notice that all isolated fixed points of the action must be critical points of $f$.

A fixed point $p \in M^{S^{1}}$ is nondegenerate if the Hessian of $f$ at $p$ is nondegenerate. An invariant version of the Morse lemma says that for every nondegenerate fixed point there exists a standard chart $(U, \phi)$ at $p$, so that on $U$

$$
f=\phi^{*}\left(f(p)-\left|z_{1}\right|^{2}-\cdots-\left|z_{\lambda}\right|^{2}+\left|z_{\lambda+1}\right|^{2}+\cdots+\left|z_{n}\right|^{2}\right) .
$$

The chart $(U, \phi)$ is called a standard chart of $f$ at $p$, and the index $\sigma(p)$ of $p$ is defined to be $2 \lambda$.

A circle orbit $s \subset C$ rit $(f)$ will be called a critical circle of $f$. Choose an invariant neighborhood $\tilde{U}$ of $s$ on which $S^{1}$ acts freely and let $\pi$ be the map $\tilde{U} \rightarrow \tilde{U} / S^{1}$. Then $s$ is nondegenerate if the Hessian of $\pi_{*}(f)$ is nondegenerate at $s / S^{1}$. The Morse lemma states that there exists an invariant neighborhood $U \subset \tilde{U}$ of $s$ and coordinates $\left(x_{1}, \ldots, x_{2 n-1}\right)$ on $U / S^{1}$, such that

$$
f=\pi^{*}\left(f(p)-x_{1}^{2}-\cdots-x_{\lambda}^{2}+x_{\lambda+1}^{2}+\cdots+x_{2 n-1}^{2}\right) .
$$


We say that $U$ together with the coordinates $x_{1}, \ldots, x_{2 n-1}$ is a standard chart of $f$ at $s$. The index $\sigma(s)$ of $s$ is defined to be $\lambda$.

Define $f$ to be an invariant Morse function if each connected component of $\operatorname{Crit}(f)$ is either a nondegenerate fixed point or a nondegenerate critical circle.

Assume $f$ is an invariant Morse function on $M$. An invariant vector field $\xi$ on $M$ is a gradient-like vector field if

1) $\xi(f)<0$ on $M-\operatorname{Crit}(f)$,

2) for every nondegenerate critical point $p$, there exists a standard chart $(U, \phi)$ of $f$ at $p$, such that $\xi$ on $U$ has coordinates

$$
\left(x_{1}, y_{1}, \ldots, x_{\lambda}, y_{\lambda},-x_{\lambda+1},-y_{\lambda+1}, \ldots,-x_{n},-y_{n}\right) .
$$

3) for every nondegenerate critical circle $s$ there exists a standard chart of $f$ at $s$, such that the pushforward vector field $\pi_{*}(\xi)$ on $U / S^{1}$ has coordinates

$$
\left(x_{1}, \ldots, x_{\lambda},-x_{\lambda+1}, \ldots,-x_{2 n-1}\right) .
$$

A gradient-like vector field $\xi$ defines a smooth flow $\phi_{\xi}(t)$ on $M$. For $q \in \operatorname{Crit}_{c}(f)$ define

$$
W_{q}^{ \pm}=\left\{x \in M \mid \lim _{t \rightarrow \pm \infty} \phi_{\xi}(t) \in q\right\}
$$

The manifold $W_{q}^{+}$is the stable manifold of $q$, and $W_{q}^{-}$is the unstable manifold of $q$. The pair $(f, \xi)$ is called an invariant Morse-Smale function on $M$ if every stable manifold intersects every unstable manifolds transversely.

Theorem 2.1. Every semi-free circle action on a compact manifold with isolated fixed points has an invariant Morse-Smale function.

The key step in the proof of our main result is a new cancellation theorem. To state it, let us introduce some notation. For a subset $I$ of $\mathbb{R}$ and a submanifold $N$ of $M$, define $N_{I}(f)=f^{-1}(I) \cap N$. When the context is clear, we will drop $f$ from the notation and set $N_{I}=$ $N_{I}(f)$. For example, for $a \in \mathbb{R}, W_{q, a}^{+}=W_{q}^{+} \cap f^{-1}(a), W_{q, \leq a}^{-}=W_{q}^{-} \cap$ $f^{-1}((-\infty, a])$, or for $a<b, M_{[a, b]}=f^{-1}([a, b])$.

Assume $V$ is an oriented manifold with a free $S^{1}$ action and that $N$ and $N^{\prime}$ are two submanifolds of $V$ which intersect transversely with $\operatorname{dim} N+\operatorname{dim} N^{\prime}=\operatorname{dim} V+1$. Also assume $N$ and the normal bundle $\nu\left(N^{\prime}\right)$ are oriented. In Section 4 we define the intersection number $N \cdot N^{\prime}$ by counting the number of circles in $N \cap N^{\prime}$ with appropriate signs. Orientations on $N$ and $\nu(N)$ are compatible with respect to an 
orientation on $V$, if at any point of $N$ a positively oriented frame on $N$ followed by a positively oriented frame on $\nu(N)$ gives a positively oriented frame on $V$. If the orientation on $N$ and $\nu(N)$ as well as on $N^{\prime}$ and $\nu\left(N^{\prime}\right)$ are compatible to an orientation on $V$, then $N \cdot N^{\prime}= \pm N^{\prime} \cdot N$.

Theorem 2.2. (The cancellation theorem.) Assume a circle action on a compact manifold $M$ is semi-free and $(f, \xi)$ is an invariant MorseSmale function on $M$. Assume $a<b$ and $M_{(a, b)}$ contains one fixed point $p$ of index $\lambda$ and one critical circle $s$ of index $\lambda+1$. Let $f(p)<c<f(s)$. Suppose $V=M_{c}$ is oriented and $N=W_{p, c}^{+}, \nu(N)$ as well as $N^{\prime}=W_{s, c}^{-}$, $\nu\left(N^{\prime}\right)$ are equipped with compatible orientations and $N \cdot N^{\prime}= \pm 1$. Further assume that either $\operatorname{dim} M \geq 8, M_{a} / S^{1}$ is simply-connected, and $M_{a}$ is connected; or $\lambda=0$; or $\lambda=\operatorname{dim} M-2$.

Then there exist a new invariant Morse function $f^{\prime}$ and a new gradientlike vector field $\xi^{\prime}$ identical to $(f, \xi)$ outside $M_{(a, b)}$ such that $M_{(a, b)}$ contains no critical circles of $f^{\prime}$ and the index of the fixed point $p$ is $\lambda+2$.

A circle action is torsion-free if the equivariant cohomology $H_{S^{1}}^{*}(M)=H_{S^{1}}^{*}(M, \mathbb{Z})$ is torsion-free as a $\mathbb{Z}$ module. For a torsion-free $S^{1}$ action define its Poincare polynomial by

$$
\mathcal{P}_{S^{1}}(M)=\sum_{k \in \mathbb{Z}_{\geq 0}} \operatorname{dim}\left(H_{S^{1}}^{k}(M)\right) t^{k} .
$$

For a fixed point $p$ define $\mathcal{M}(p)=\frac{t^{\sigma(p)}}{1-t^{2}}$, and for a critical circle $s$ set $\mathcal{M}(s)=t^{\sigma(s)}$. Define the Morse polynomial of an invariant Morse function $f$ by

$$
\mathcal{M}_{S^{1}}(M, f)=\sum_{q \in C r i t_{c}(f)} \mathcal{M}(q) .
$$

An invariant Morse function is perfect if the Poincare and Morse polynomials are the same: $\mathcal{P}_{S^{1}}(M)=\mathcal{M}_{S^{1}}(M, f)$. We have given all the definitions needed to state Theorem 1.1.

\section{Existence of invariant Morse-Smale functions}

We believe many results of this section are known to the experts in the field (see [3] for further references). We provide the proofs of these results for completeness.

Theorem 3.1. Every semi-free circle action on a compact manifold with isolated fixed points has an invariant Morse function. 
Proof. Let $M^{S^{1}}=\left\{p_{1}, \ldots, p_{m}\right\}$. Around every fixed point $p_{i}$ there exists a standard coordinate chart $\left(U_{i}, \phi_{i}\right)$. Without loss of generality, we can assume each $U_{i}$ is diffeomorphic to a unit ball $B_{1}$ inside of $\mathbb{C}^{n}$ and that $U_{i}$ 's are pairwise disjoint.

Define $f=\phi_{i}^{*}\left(|z|^{2}\right)$ on every $U_{i}$. We want to extend $f$ to $M-U$ , where $U=\cup_{i} U_{i}$. The manifold $X=(M-U) / S^{1}$ is compact and $\partial X=(\partial \bar{U}) / S^{1}$. By [8, Theorem 2.5] there exists a Morse function $h:(X, \partial X) \rightarrow([1, \infty), 1)$. Denote by $\pi$ the projection $M-U \rightarrow X$. Define $f=\pi^{*}(h)$ on $M-U$. Then $f$ is smooth outside of $\partial \bar{U}$.

To finish the proof use the following smoothing argument. Choose an invariant tubular neighborhood $V$ of $\partial \bar{U}$ and identify it with $(-\varepsilon, \varepsilon) \times$ $\partial \bar{U}$. Let $f^{-}, f^{+}$be two smooth functions on $V$, such that $f^{-}(t, x)=$ $f(t, x)$ for $(t, x) \in(-\varepsilon, 0) \times \partial \bar{U}$ and $f^{+}(t, x)=f(t, x)$ for $(t, x) \in$ $(0, \varepsilon) \times \partial \bar{U}$. Without loss of generality, we can assume $\frac{\partial f^{-}}{\partial t}(t, x)>0$ and $\frac{\partial f^{+}}{\partial t}(t, x)>0$ on $V$ and $f^{+}(t, x)>f^{-}(t, x)$ for $|t|>\varepsilon / 2$. Let $\mu(t)$ be a smooth positive nondecreasing function, with $\mu(t)=0$, for $t \leq-\varepsilon ; \mu(t)=1$ for $t \geq \varepsilon$; and $\mu(t)=\frac{1}{2}$ for $|t|<\varepsilon / 2$. Change $f$ to $\mu f^{+}+(1-\mu) f^{-}$on $V$. Then $f$ is smooth on $M$. An easy computation shows that $\frac{\partial f}{\partial t}>0$ on $V$ which implies that this construction does not create new critical points of $f$.

Every invariant Morse function together with an invariant gradientlike vector field on a manifold with isolated fixed points defines a partial order on $\operatorname{Crit}_{c}(f)$ as follows. If $q_{1}, q_{2} \in \operatorname{Crit}_{c}(f)$, set $q_{1} \prec q_{2}$ if $q_{1}$ is in the closure of $W_{q_{2}}^{-}$. Complete this relation to satisfy transitivity and hence define a partial order on $\operatorname{Crit}_{c}(f)$. A map $c: \operatorname{Crit}_{c}(f) \rightarrow \mathbb{R}$ is said to be order preserving if $q_{1} \prec q_{2}$ implies $c\left(q_{1}\right)<c\left(q_{2}\right)$.

Theorem 3.2. Assume a circle action on $M$ is semi-free and has isolated fixed points. Let $f$ be an invariant Morse function and $\xi$ be its gradient-like vector field on $M$, then every order preserving map on $\mathrm{Crit}_{c}(f)$ can be extended to an invariant Morse function.

Proof. Throughout the proof $\xi$ and the partial order $\prec$ are fixed. Notice that for every $q \in \mathrm{Crit}_{c}(f)$ there exists an invariant Morse function $g$, such that $g=f$ outside a small invariant neighborhood $U$ of $q, g(q)=$ $f(q)+\varepsilon$ for a small enough $\varepsilon$, and $\xi$ is an invariant gradient-like vector field for $g$. The function $g$ can be defined by $g=f+\rho$, where $\rho$ is an invariant "bump" function, such that $\rho=0$ outside of $U, \rho=\varepsilon$ on a compact set $K \subset U$ and $|\xi(\rho)|<|\xi(f)|$ on $U-K$. 
Hence the theorem follows from the following statement. Let $f$ have two critical values in the interval $(a, b)$, such that one critical level contains $\left\{q_{1}, \ldots, q_{k}\right\} \subset \operatorname{Crit}_{c}(f)$ and the other contains $\left\{r_{1}, \ldots, r_{m}\right\} \subset$ $\mathrm{Crit}_{c}(f)$. Moreover, assume non of the $q_{i}$ 's are compatible with the $r_{j}$ 's in the partial order $\prec$, that is stable and unstable manifolds of $q_{i}$ 's and $r_{j}$ 's never intersect. Then for any $a_{1}, a_{2} \in(a, b)$, there exists an invariant Morse function $g$, such that $\xi$ is an invariant gradient-like vector field of $g, f=g$ outside $M_{[a, b]}$, the set of critical points of $g$ is the same as the set of critical points of $f$, and $g\left(q_{i}\right)=a_{1}, g\left(r_{j}\right)=a_{2}$.

Without the word "invariant" the above statement is [8, Theorem 4.2]. This allows to give a proof of this statement by making a few minor changes in the proof of [ 8 , Theorem 4.2], mainly by inserting the word "invariant" where necessary.

Let $S^{1}$ act on two manifolds $M$ and $N$. An invariant isotopy between two invariant diffeomorphisms $h_{0}, h_{1}: M \rightarrow N$ is a smooth map $h$ : $M \times[0,1] \rightarrow N$, such that $h_{0}(x)=h(x, 0), h_{1}(x)=h(x, 1)$ and every $h_{t}(x)=h(x, t)$ is an invariant diffeomorphism. We say that $h$ is an isotopy of identity if $M=N$ and $h_{0}$ is an identity map.

Lemma 3.3. Assume we are given an invariant Morse function $f$ on $M$ with an invariant gradient-like vector field $\xi$, a non-critical level $M_{a}=f^{-1}(a)$, and a diffeomorphism $h_{1}: M_{a} \rightarrow M_{a}$ that is invariantly isotopic to the identity. If for $a<b$ the interval $[a, b]$ contains no critical values, then it is possible to construct a gradient-like vector field $\bar{\xi}$, such that $\xi=\bar{\xi}$ outside $M_{[a, b]}$ and $\bar{\varphi}=h_{1} \circ \varphi$, where $\varphi$ and $\bar{\varphi}$ are the diffeomorphisms $M_{b} \rightarrow M_{a}$ determined by following the trajectories of $\xi$ and $\bar{\xi}$ respectively.

Proof. This lemma is the invariant version of [8, Lemma 4.7] whose proof can be modified (mostly by inserting the word "invariant") to provide a proof of the lemma.

Proof of Theorem 2.1. Using Theorem 3.1 choose an invariant Morse function $f$ on $M$. Pick any invariant riemmannian metric on $M$, let $\nu=-\operatorname{grad}(f)$. On canonical charts $U_{i}$ of $f$ at every fixed point or critical circle define a vector field $\mu$ which is a gradient-like vector field on $\cup U_{i}$. Pick a nonnegative function $\rho$ which is zero outside of $\cup U_{i}$ and one in a neighborhood of $\operatorname{Crit}(f)$. Then $\xi=\rho \mu+(1-\rho) \nu$ is a gradient-like vector field.

By Theorem 3.2, we can assume that every critical level of $f$ contains exactly one fixed point or one critical circle. Let $c_{1}<\cdots<c_{k}$ be the 
critical values of $f$ and $q_{1}, \ldots, q_{k} \in \operatorname{Crit}_{c}(f)$ with $f\left(q_{i}\right)=c_{i}$. By induction on $i$ we will prove that there exists an invariant gradient-like vector field $\xi$ of $f$, such that every $W_{q_{j}}^{-}$intersects transversely with every stable manifold for any $j \leq i$.

The base of the induction is trivial for $i=1$. Assume our induction statement holds for $i-1$. To prove the theorem it is enough to perturb $\xi$ on the preimage of $\left(c_{i-1}, c_{i}\right)$ to guarantee that $W_{q_{i}}^{-}$intersects transversely with all the stable manifolds.

Choose a number $a$ between $c_{i-1}$ and $c_{i}$. There exists an invariant tubular neighborhood $U$ of $W_{q_{i}, a}^{-}$inside $M_{a}$ which is diffeomorphic to an $\mathbb{R}^{k}$ vector bundle $\pi: V \rightarrow W_{q_{i}, a}^{-}$. If $q_{i}$ is a critical circle, then $V$ can be chosen to be an $S^{1}$-trivial bundle, that is $U$ is invariantly diffeomorphic to $W_{q_{i}, a}^{-} \times \mathbb{R}^{k}$, with $S^{1}$ acting only on the first component. Let $P: U \rightarrow \mathbb{R}^{k}$ be the natural projection. Let $Y_{j}$ be the set of critical values of $P$ restricted to $W_{q_{j}, a}^{+} \cap U$ and set $Y=\cup_{j} Y_{j}$. By Sard's theorem $Y$ has measure zero inside $\mathbb{R}^{k}$. Choose $v \in \mathbb{R}^{k}-Y$. There exists an invariant isotopy of identity $h_{t}$ on $U \cong W_{q_{i}, a}^{-} \times \mathbb{R}^{k}$, such that $h_{1}\left(W_{q_{i}, a}^{-} \times 0\right)=W_{q_{i}, a}^{-} \times v$ and $h_{t}$ is the identity outside some compact neighborhood $K \subset U$ of $W_{q_{i}, a}^{-}$. Extend this isotopy to $M_{a}$ by setting it to be identity outside of $U$. Then apply Lemma 3.3 to perturb the gradient-vector field $\xi$ to guarantee that the induction assumption is satisfied.

If $q_{i}$ is a fixed point, then $\pi: V \rightarrow W_{q_{i}, a}^{-}$is no longer $S^{1}$-trivial. But $W_{q_{i}, a}$ can be covered by finitely many compact invariant sets $\left\{K_{\ell}\right\}_{\ell=1}^{L}$ such that each $K_{\ell}$ is inside an open invariant set $W_{\ell}$ which invariantly contracts to a circle. Without loss of generality we may assume that $W_{\ell}$ 's are invariantly diffeomorphic to $B_{1}^{\circ} \times S^{1}$ (where $B_{1}^{\circ}$ is an open ball of radius 1 and $S^{1}$ acts only on the second factor) and $K_{\ell}$ 's are $B_{\frac{1}{2}} \times S^{1}$ inside $W_{\ell}$. Set $V_{\ell}=\pi^{-1}\left(W_{\ell}\right)$, then the bundle $V_{\ell} \rightarrow W_{\ell}$ can be trivializes and, in particular, each $V_{\ell}$ is invariantly diffeomorphic to $W_{\ell} \times \mathbb{R}^{k}$ with $S^{1}$ acting only on the first factor.

Let $P_{1}$ be the natural projection from $V_{1}$ to $\mathbb{R}^{k}$. Assume $v_{1}$ is a regular value for every restriction of $P_{1}$ to $W_{q_{j}, a}^{+}$. Then construct an isotopy of identity $h_{t}^{1}$ on $M_{a}$ which is identity outside $V_{1}$ and such that $h_{1}^{1}$ takes $K_{1} \times 0$ onto $K_{1} \times v_{1}$. This will guarantee that $h_{1}^{1}\left(W_{q_{i}, a}^{-}\right)$is transverse to every $W_{q_{j}, a}^{+}$on $K_{1}$.

To construct an isotopy of identity $h_{t}^{2}$, choose a trivialization $h_{1}^{1}\left(V_{2}\right)=h_{1}^{1}\left(W_{2}\right) \times \mathbb{R}^{k}$, let $P_{2}$ be the natural projection onto the second factor, and choose $v_{2}$ which is a regular value for every restriction 
of $P_{2}$ to $W_{q_{j}, a}^{+}$. Choose $h_{t}^{2}$, such that it is identity outside of $h_{1}^{1}\left(V_{2}\right)$ and $h_{1}^{2}$ takes $h_{1}^{1}\left(K_{2}\right) \times 0$ onto $h_{1}^{1}\left(K_{2}\right) \times v_{2}$. Since $K_{1}$ is compact and transversality is an open condition we can choose $v_{2}$ to be small enough so that $h_{1}^{2}\left(h_{1}^{1}\left(W_{q_{i}, a}^{-}\right)\right)$is transverse to $W_{q_{j}, a}^{+}$on $K_{1}$ Then $h_{1}^{2}\left(h_{1}^{1}\left(W_{q_{i}, a}^{-}\right)\right)$ is transverse to all $W_{q_{j}, a}^{+}$on $K_{1} \cup K_{2}$.

This process can be continued to construct invariant isotopies of identity $h_{t}^{m}$, such that $h_{1}^{m}\left(\ldots\left(h_{1}^{1}\left(W_{q_{i}, a}^{-}\right)\right) \ldots\right)$ is transverse to every $W_{q_{j}, a}^{+}$on $\cup_{\ell=1}^{m} K_{\ell}$. Eventually we will construct $h_{t}^{1}, \ldots, h_{t}^{L}$ such that $h_{1}^{L}\left(\ldots\left(h_{1}^{1}\left(W_{q_{i}, a}^{-}\right)\right) \ldots\right)$ is transverse to every $W_{q_{j}, a}^{+}$. Repeatedly apply Lemma 3.3 to perturb $\xi$ to a new gradient-like vector field to guarantee $W_{q_{i}}^{-}$intersects all stable manifolds transversely.

Minor modifications of the above argument provide a slightly stronger result.

Theorem 3.4. Assume a circle action on a compact manifold $M$ is semi-free and has isolated fixed points. Then for every Morse function $f$ there exists a gradient-like vector field $\xi$ such that $(f, \xi)$ is MorseSmale.

Remark 3.5. As mentioned in the introduction, it is not true that any compact manifold with a circle action has an invariant Morse-Smale function.

\section{Cancellation Theorems.}

The following example is an illustration to the cancellation theorems.

Example 4.1. Let $S^{1}$ act on $\mathbb{R}^{2}=\mathbb{C}$ with weight 1 . Consider a function $f(z)=v(|z|)$ where $v(r)$ is a real valued function which is equal to $r^{2}$ near zero, strictly increases as $r$ increases from 0 to 1 , equals to $2 r-r^{2}$ near 1 , and strictly decreases as $r$ goes from 1 to infinity. Then $f$ is an invariant Morse function with a fixed point at the origin of index 0 and a critical circle at the unit circle of index 1 . An invariant gradient-like vector field is given by $(x \rho(|z|), y \rho(|z|))$ at the point $z=x+\sqrt{-1} y$, where $\rho(r)=-1$ near $0, \rho(r)<0$ when $0<r<1, \rho>0$ when $r>1$, and $\rho=\frac{1-r}{r}$ near 1 .

Changing $f$ to $2-|z|^{2}$ and the gradient-like vector field to $(x, y)$ cancels the critical circle and increases the index of the origin to 2. See Figure 1.

Theorem 4.2. (The preliminary cancellation theorem) Assume $f$ is an invariant Morse function on a compact manifold $M$ with semi-free 
circle action and $\xi$ is a gradient-like vector field on $M$. Assume $a<b$ and $M_{(a, b)}$ contains a single fixed point $p$ of index $\lambda$ and a single critical circle $s$ of index $\lambda+1$. Let $T=W_{p}^{+} \cap W_{s}^{-}$be a single disc. Then there exists an invariant Morse function $f^{\prime}$ identical to $f$ outside $M_{(a, b)}$, such that $M_{(a, b)}$ contains no critical circles of $f^{\prime}$ and the index of the fixed point $p$ is $\lambda+2$.

Proof. Let $S^{1}$ act on $\mathbb{C}^{n}$ with weight $(1, \ldots, 1)$. Define

$$
\begin{gathered}
h\left(z_{1}, \ldots, z_{n}\right)= \\
f(p)+(f(s)-f(p)) v\left(\left|z_{1}\right|\right)-\left|z_{2}\right|^{2}-\cdots-\left|z_{\lambda+1}\right|^{2}+\left|z_{\lambda+2}\right|^{2}+\cdots+\left|z_{n}\right|^{2},
\end{gathered}
$$

where $v$ is the function from Example 4.1. Moreover, let $\eta$ be the vector field given by

$\left.\left(x_{1} \rho\left(\left|z_{1}\right|\right), y_{1} \rho\left(\left|z_{1}\right|\right), x_{2}, y_{2}, \ldots, x_{\lambda+1}, y_{\lambda+1},-x_{\lambda+2},-y_{\lambda+2}, \ldots,-x_{n},-y_{n}\right)\right)$,

where $\rho$ is the function from Example 4.1. Denote by $\tilde{p}$ the origin of $\mathbb{C}^{n}$ and by $\tilde{s}$ the unit circle inside $\mathbb{C} \times 0 \times \cdots \times 0$.

Claim 4.3. There exists an invariant diffeomorphism $g$ which maps an invariant neighborhood $V$ of the disc $D=\left\{\left(z_{1}, 0, \ldots, 0\right) ;\left|z_{1}\right| \leq 1\right\}$ onto an invariant neighborhood $U$ of $T$ with $g^{*}(f)=h$ and $g_{*}(\eta)=\xi$.

Proof of Claim 4.3. There exist diffeomorphisms $g_{p}$ and $g_{s}$ from neighborhoods $V_{\tilde{p}}$ of $\tilde{p}$ and $V_{\tilde{s}}$ of $\tilde{s}$ onto neighborhoods $U_{p}$ of $p$ and $U_{s}$ of $s$ which take $D$ onto $T$. Let $\varepsilon$ be small enough so that $f(p)+\varepsilon<f(s)-\varepsilon$. Then set $g$ equal to $g_{p}$ and to $g_{s}$ outside $M_{[f(p)+\varepsilon, f(s)-\varepsilon]}$. By following the trajectories of $\xi$ and $\eta$ the map $g$ extends to $D$.

Let $L_{\tilde{p}}=V_{\tilde{p}, f(p)+\varepsilon}$ (recall our conventions $V_{\tilde{p}, f(p)+\varepsilon}=V_{\tilde{p}} \cap h^{-1}(f(p)+$ $\varepsilon))$ and $L_{\tilde{s}}=V_{\tilde{s}, f(s)-\varepsilon}$. Set $D_{\tilde{p}}=D \cap h^{-1}(f(p)+\varepsilon)$ and $D_{\tilde{s}}=D \cap$ $h^{-1}(f(s)-\varepsilon)$. By following the trajectories of $\eta$ we can define an invariant diffeomorphism $j_{1}$ from a neighborhood $W_{\tilde{s}}$ of $D_{\tilde{s}}$ inside $L_{\tilde{s}}$ onto a neighborhood $W_{\tilde{p}}$ of $D_{\tilde{p}}$ inside $L_{\tilde{p}}$. At the same time, if we apply $g_{s}$, then follow the trajectories of $\xi$ and then apply $g_{p}^{-1}$ we get another diffeomorphism $j_{2}$ from a neighborhood $W_{\tilde{s}}^{\prime}$ of $D_{\tilde{s}}$ inside $L_{\tilde{s}}$ onto a neighborhood $W_{\tilde{p}}^{\prime}$ of $D_{\tilde{p}}$ inside $L_{\tilde{p}}$.

The invariant diffeomorphisms $j_{1}$ and $j_{2}$ define diffeomorphisms $\bar{j}_{1}$ and $\bar{j}_{2}$ of $W_{\tilde{s}} / S^{1}$ to $W_{\tilde{p}} / S^{1}$ and of $W_{\tilde{s}}^{\prime} / S^{1}$ to $W_{\tilde{p}}^{\prime} / S^{1}$. Using $[8$, Theorem 5.6] we can construct an isotopy of identity $\bar{\psi}_{t}$ of a neighborhood $W / S^{1}$ of $D_{\tilde{p}} / S^{1}$ inside $L_{\tilde{p}} / S^{1}$ which intertwines $\bar{j}_{1}$ and $\bar{j}_{2}$, that is $\bar{\psi}_{1}\left(\bar{j}_{1}\right)=\bar{j}_{2}$. Since we can assume $W / S^{1}$ is contractible, there exists an 
identification $W=S^{1} \times W / S^{1}$ and hence a lift of $\bar{\psi}_{t}$ to an invariant isotopy $\psi_{t}$ which intertwines $j_{1}$ and $j_{2}$ on $W$. Since $\psi_{t}$ is identity outside some compact set the isotopy $g_{p}\left(\psi_{t}\right)$ extends to the whole $M_{f(p)+\varepsilon}$.

Use Lemma 3.3 to perturb $\xi$ on $M_{[f(p)+\varepsilon, f(s)-\varepsilon]}$ to guarantee that $j_{1}=j_{2}$ for the newly constructed gradient-vector field. The claim is proved, since $g$ extends to an open neighborhood of $D$ by following the trajectories of $\xi$ and $\eta$.

The next step in the proof of Theorem 4.2 is to change $\xi$ to $\xi^{\prime}$ inside $U$ to guarantee that there is only one critical point of $\xi^{\prime}$ inside $M_{[a, b]}$.

By an argument identical to the proof of Assertion 1 in the proof of [8, Theorem 5.4], there exists an invariant neighborhood $U^{\prime \prime}$ of $T$ inside an invariant neighborhood $U^{\prime}$ of $T$ such that $\overline{U^{\prime}} \subset U$ and no trajectory of $\xi$ enters $U^{\prime \prime}$ then exits $U^{\prime}$ and then returns to $U^{\prime \prime}$.

Change $\xi$ to $\xi^{\prime}$, such that $\xi=\xi^{\prime}$ outside of $U^{\prime \prime}$, while inside $U^{\prime \prime}$

$\left.\xi^{\prime}=g_{*}\left(v_{1}\left(x_{1}, \tau\right), v_{2}\left(y_{1}, \tau\right), x_{2}, y_{2}, \ldots, x_{\lambda+1}, y_{\lambda+1},-x_{\lambda+2},-y_{\lambda+2}, \ldots,-x_{n},-y_{n}\right)\right)$,

where $\tau=\left|z_{2}\right|^{2}+\cdots+\left|z_{n}\right|^{2}, v_{1}\left(x_{1}, 0\right)=x_{1}, v_{2}\left(y_{1}, 0\right)=y_{1}$. Moreover, $v_{1}$ and $v_{2}$ are chosen such that $\xi^{\prime}=\xi$ outside a compact neighborhood of $T$ inside $U^{\prime \prime}$. Clearly, $\xi^{\prime}$ has only one critical point, which is $p$.

Let us show that there is no trajectory $\psi(t, x)$ of $\xi^{\prime}$ through $x$ which lies completely inside $U^{\prime}$. Let $x=g\left(x_{1}^{\circ}, y_{1}^{\circ}, \ldots, x_{n}^{\circ}, y_{n}^{\circ}\right)$. If one of the coordinates $x_{2}^{\circ}, y_{2}^{\circ}, \ldots, x_{n}^{\circ}, y_{n}^{\circ}$ is not zero, then this coordinate of $\psi(t, x)$ must increase or decrease exponentially and hence this trajectory eventually exits $U^{\prime}$ either as $t$ increases or decreases. Otherwise, if only $x_{1}$ or $y_{1}$ are not zero, then again the trajectory will exit $U^{\prime}$ as $t$ increases, since $\xi^{\prime}\left(x_{1}, y_{1}, 0, \ldots, 0\right)=c\left(x_{1}, y_{1}, 0, \ldots, 0\right)$ for some positive $c$.

We now show $\xi^{\prime}$ has no periodic trajectories and any trajectory is inside $W_{p}^{+}$or $W_{p}^{-}$, or connects $M_{b}$ to $M_{a}$ (at this point we forget about $\xi$, so that $W_{p}^{+}$and $W_{p}^{-}$are the stable and unstable manifolds of $p$ of $\left.\xi^{\prime}\right)$. Indeed, if a trajectory of $\xi^{\prime}$ does not intersect $U^{\prime \prime}$, it connects $M_{b}$ to $M_{a}$, since it coincides with a trajectory of $\xi$. If, on the other hand, a trajectory $\psi(t, x)$ of $\xi^{\prime}$ through a point $x$ inside $U^{\prime \prime}$ intersects $U^{\prime}$ and is not inside $W_{p}^{-}$or $W_{p}^{+}$, then there should be two nonzero coordinates of $x$ of indices $i, j$ with $i \leq \lambda+1<j$. Such a trajectory must exit $U^{\prime}$ as $t$ increases and as $t$ decreases and never return to $U^{\prime}$. Hence this trajectory connects $M_{a}$ to $M_{b}$. This finishes the construction of $\xi^{\prime}$.

It remains to construct an invariant Morse function for which $\xi^{\prime}$ is a gradient-like vector field. To do this we will define an invariant Morse function $f^{\prime}$ on $M_{[a, b]}$ with only one critical point $p$ and with $f^{\prime}\left(M_{a}\right)=a$, 
$f^{\prime}\left(M_{b}\right)=b$ and $f(p)=c$ for $a<c<b$. Then set $f^{\prime}=f$ outside of $M_{[a, b]}$ and, by the smoothing procedure described in the proof of Theorem 3.1 , smoothen $f^{\prime}$ in a neighborhood of $M_{a}$ and $M_{b}$.

Let $V$ be an invariant neighborhood of $p$ inside a canonical chart of $\xi^{\prime}$. Choose $\varepsilon$ with $a<c-\varepsilon$ and $c+\varepsilon<b$. We can clearly define $f^{\prime}$ on $V_{[c-\varepsilon, c+\varepsilon]}$.

Choose invariant neighborhoods $V^{+}, V^{-}$of $W_{p}^{+}, W_{p}^{-}$which are preserved by the flow of $\xi^{\prime}$. Then $f^{\prime}$ can be define on $V_{[c+\varepsilon, b]}^{+}$and $V_{[a, c-\varepsilon]}^{-}$. Using again the smoothing argument $f^{\prime}$ can be defined on a certain neighborhood $W$ of $W_{p}^{+} \cup W_{p}^{-}$. Since there are no critical points on $M_{[a, b]}-\left(W_{p}^{+} \cup W_{p}^{-}\right)$, there exists an invariant function $g$ on $M_{[a, b]}-$ $\left(W_{p}^{+} \cup W_{p}^{-}\right)$with $\xi^{\prime}(g)<0, g\left(M_{a}\right)=a$ and $g\left(M_{b}\right)=b$.

Let $\mu$ be an invariant function on $M_{a}$ which is equal to 0 outside $M_{a} \cap V^{-}$and equal to 1 in a neighborhood of $M_{a} \cap W_{p}^{-}$. By following the trajectories of $\xi^{\prime}$ we get a map $M_{[a, b]}-\left(W_{p}^{+} \cup W_{p}^{-}\right) \rightarrow M_{a}-W_{p}^{-}$. Pull back $\mu$ to a function $\nu$ on $M_{[a, b]}-\left(W_{p}^{+} \cup W_{p}^{-}\right)$, extend it to $M_{[a, b]}$ by setting $\nu=1$ on $W_{p}^{+} \cup W_{p}^{-}$. Then the function $\nu f^{\prime}+(1-\nu) g$ is the invariant Morse function on $M_{[a, b]}$ we are looking for.

Let $S^{1}$ act freely on a manifold $V$. Assume two invariant submanifolds $N$ and $N^{\prime}$ of dimensions $r+1$ and $s+1$ intersect transversely and $r+s=\operatorname{dim} V-1$. Then $N$ and $N^{\prime}$ intersect along some circles $s_{1}, \ldots, s_{k}$. Assume $N$ and the normal bundle $\nu\left(N^{\prime}\right)$ are oriented. At a point $x_{i} \in s_{i}$ choose a positively oriented frame $\xi_{0}, \ldots, \xi_{r}$ on $N$ such that $\xi_{0}$ is the restriction to $x_{i}$ of the infinitesimal vector field associated to the circle action on $s_{i}$. Since $N$ and $N^{\prime}$ intersect transversely, $\xi_{1}, \ldots, \xi_{r}$ form a basis of the normal bundle to $N^{\prime}$ at $x_{i}$. The intersection number of $N$ and $N^{\prime}$ at $s_{i}$ is defined to be +1 if $\xi_{1}, \ldots, \xi_{r}$ is a positively oriented basis and -1 otherwise. This definition does not depend on the choice of the point $x_{i}$ inside of $s_{i}$. The intersection number $N \cdot N^{\prime}$ of $N$ and $N^{\prime}$ is the sum of all the intersection numbers at the circles $s_{i}$.

If $V$ is oriented, then we say that orientations on $N$ and on $\nu(N)$ are compatible with respect to the orientation on $V$ if at any point on $N$ a positively oriented frame on $N$ followed by a positively oriented frame on $\nu(N)$ gives a positively oriented frame on $V$. If compatible orientation on $N$ and $\nu(N)$ as well as on $N^{\prime}$ and $\nu\left(N^{\prime}\right)$ are used to define $N \cdot N^{\prime}$ and $N^{\prime} \cdot N$ then $N \cdot N^{\prime}= \pm N^{\prime} \cdot N$ depending on parity of $r s$. 
Before we can prove the cancellation theorem we need two preliminary results:

Theorem 4.4. Let $N$ and $N^{\prime}$ be smooth closed invariant transversely intersecting submanifolds of dimensions $r+1$ and $s+1$ in the $(r+s+1)$ manifold $V$ on which $S^{1}$ acts freely. Suppose $N$ and the normal bundle to $N^{\prime}$ are oriented. Assume $r+s \geq 5, s \geq 3$ and, in case $r=2$, suppose the inclusion map $\pi_{1}\left(\left(V-N^{\prime}\right) / S^{1}\right) \rightarrow \pi_{1}\left(V / S^{1}\right)$ is injective.

Let $s_{1}, s_{2} \in N \cap N^{\prime}$ be circles with opposite intersection numbers. Assume there exist two invariant embeddings of $S^{1} \times[0,1]$ into $N$ and into $N^{\prime}$ which connect $s_{1}$ to $s_{2}$, such that both embeddings miss $N \cap$ $N^{\prime}-\left\{s_{1}, s_{2}\right\}$. The composition of two embeddings provides a map of $S^{1} \times S^{1}$ into $V$. Assume $S^{1} \times S^{1}$ is invariantly contractible to a circle inside $V$.

With these assumptions there exists an invariant isotopy of identity $h_{t}$ on $V$, such that $h_{t}$ is identity near $N \cap N^{\prime}-\left\{s_{1}, s_{2}\right\}$ and $h_{1}(N) \cap N^{\prime}=$ $N \cap N^{\prime}-\left\{s_{1}, s_{2}\right\}$.

Proof. We deliberately stated this theorem in the form very similar to [8, Theorem 6.6]. Actually, [8, Theorem 6.6], applied to manifolds $V / S^{1}, N / S^{1}$, and $N^{\prime} / S^{1}$ provides an isotopy $\tilde{h}_{t}$ of $V / S^{1}$ which is identity near $N / S^{1} \cap N^{\prime} / S^{1}-\left\{s_{1} / S^{1}, s_{2} / S^{1}\right\}$ and such that $\tilde{h}_{1}\left(N / S^{1}\right) \cap N^{\prime} / S^{1}=$ $N / s^{1} \cap N^{\prime} / S^{1}-\left\{s_{1} / S^{1}, s_{2} / S^{1}\right\}$. So, it is enough to show that $\tilde{h}_{t}$ can be lifted to an invariant isotopy $h_{t}$ on $V$.

In the proof of $[\mathbf{8}$, Theorem 6.6$]$ the constructed isotopy $\tilde{h}_{t}$ is identity outside some contractible open set $U$ in $V$. (If $D$ is the $\operatorname{disc}$ in $V / S^{1}$, whose boundary consists of two arcs connecting $s_{1} / S^{1}$ to $s_{2} / S^{1}$, then $U$ is a neighborhood of $D$.) Set $h_{t}$ to be equal to identity outside of $\pi^{-1}(U)$ (where $\pi: V \rightarrow V / S^{1}$ ). Choosing an invariant trivialization $\pi^{-1}(U)=U \times S^{1}$ allows us to lift $\tilde{h}_{t}$ to $h_{t}$ on $U$.

Lemma 4.5. Given a semi-free circle action on a compact connected manifold $M$, let $f$ be an invariant Morse function on $M$ and $\xi$ a gradient-like vector field. For regular values $a<b$ of $f$, suppose $M_{[a, b]}$ contains exactly one critical point $p$ and $M_{a}$ is connected then

$\pi_{1}\left(M_{a} / S^{1}\right)=\pi_{1}\left(M_{b} / S^{1}\right)=\pi_{1}\left(\left(M_{a}-W_{p, a}^{-}\right) / S^{1}\right)=\pi_{1}\left(\left(M_{b}-W_{p, b}^{+}\right) / S^{1}\right)$.

Proof. Since $p$ is of even index, $M_{b}, M_{a}-W_{p, a}^{-}$and $M_{b}-W_{p, a}^{+}$are connected and it makes sense to talk about their fundamental groups. The trajectories of $\xi$ provide an invariant diffeomorphism of $M_{a}-W_{p, a}^{-}$ and $M_{b}-W_{p, b}^{+}$, in particular, $\pi_{1}\left(\left(M_{a}-W_{p, a}^{-}\right) / S^{1}\right)=\pi_{1}\left(\left(M_{b}-W_{p, b}^{+}\right) / S^{1}\right)$. 
Let $U$ be an invariant tubular neighborhood of $W_{p, a}^{-}$inside $M_{a}$. Then $\pi_{1}\left(U / S_{1}\right)=\pi_{1}\left(W_{p, a}^{-} / S^{1}\right)=1$, since $W_{p, a} / S^{1}$ is homeomorphic to a complex projective space. Moreover, $\pi_{1}\left(U-W_{p, a}^{-} / S^{1}\right)=1$, since $U-W_{p, a}^{-} / S^{1}$ is homotopy equivalent to an even sphere bundle over a complex projective space. Hence, van Kampen's theorem applied to the covering of $M_{a} / S^{1}$ by $\left(M_{a}-W_{p, a}^{-}\right) / S^{1}$ and $U / S^{1}$ immediately leads to $\pi_{1}\left(M_{a} / S^{1}\right)=\pi_{1}\left(\left(M_{a}-W_{p, a}^{-}\right) / S^{1}\right)$. Analogously, we can prove $\pi_{1}\left(M_{b} / S^{1}\right)=\pi_{1}\left(\left(M_{b}-W_{p, b}^{+}\right) / S^{1}\right)$.

Proof of Theorem 2.2. Taking into account that $\lambda$ is even, there are five different cases:

Case 1: $\lambda=0$. Then $N^{\prime}$ is two copies of a circle and $N \cdot N^{\prime}= \pm 1$ implies $N \cap N^{\prime}$ is just one circle. Hence Theorem 4.2 applies.

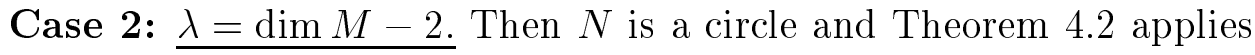
again.

Case 3: $\operatorname{dim} M-4>\lambda \geq 4, \operatorname{dim} M \geq 8, M_{a} / S^{1}$ is simply-connected, $M_{a}$ is connected. Set $r+1=\operatorname{dim} N=\operatorname{dim} M-\lambda-1$ and $s+1=$ $\operatorname{dim} N^{\prime}=\lambda+1$. Then $r+s=\operatorname{dim} M-2 \geq 5, s=\lambda \geq 3$, and $r=\operatorname{dim} M-\lambda-2 \geq 3$. Lemma 4.5 implies $\pi_{1}\left(M_{c} / S^{1}\right)=1$, hence Theorem 4.4 applies to this situation. Repeatedly apply Theorem 4.4 to find an invariant homotopy $h_{t}$ of $M_{c}$ to guarantee $h_{1}(N) \cap N^{\prime}$ is a single circle. Then we can apply Lemma 3.3 to find new $\left(f^{\prime}, \xi^{\prime}\right)$ for which $W_{p}^{+}$intersect $W_{s}^{-}$along a single disc. Finally we apply the preliminary cancellation theorem to finish this case.

Case 4: $\lambda=\operatorname{dim} M-4, \operatorname{dim} M \geq 8, M_{a} / S^{1}$ is simply-connected, $M_{a}$ is connected. Define $r+1=\operatorname{dim} N=\operatorname{dim} M-\lambda-1=3, s+1=$

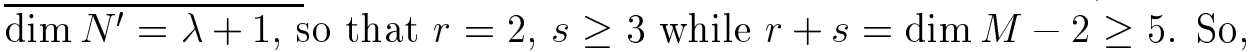
to apply Theorem 4.4 and then Theorem 4.2 as in Case 3, it is enough to show $\pi_{1}\left(\left(M_{c}-N^{\prime}\right) / S^{1}\right)=\pi_{1}\left(M_{c} / S^{1}\right)=1$. Notice $\pi_{1}\left(M_{c} / S^{1}\right)=1$ by Lemma 4.5. To show $\pi_{1}\left(\left(M_{c}-N^{\prime}\right) / S^{1}\right)=1$, identify $N^{\prime} / S^{1}$ with the sphere of dimension $\operatorname{dim} M-3$ and then use an argument analogues to the proof of Lemma 4.5 .

Case 5: $\lambda=2, \operatorname{dim} M \geq 8, M_{a} / S^{1}$ is simply-connected, $M_{a}$ is connected. Here we interchange the roles of $N$ and $N^{\prime}$ in Theorem 4.4. Namely, set $r+1=\operatorname{dim} N^{\prime}=3$ and $s+1=\operatorname{dim} N=\operatorname{dim} M-3$. Then $r=2$, $r+s \geq 5$ and $s \geq 3$, so to apply Theorem 4.4 and the preliminary cancellation theorem as in Case 3, it is enough to check $\pi_{1}\left(\left(M_{c}-N\right) / S^{1}\right)=$ $\pi_{1}\left(M_{c} / S^{1}\right)$, which immediately follows from Lemma 4.5. 


\section{Equivariant Cohomology and Morse Theory.}

This section discusses properties of equivariant cohomology and its connections to Morse theory. Let $E S^{1}$ be a contractible space with a free $S^{1}$ action. (For example, $E S^{1}$ can be the infinite dimensional sphere $S^{\infty}$.) The equivariant cohomology is defined by

$$
H_{S^{1}}^{*}(M, \mathbb{Z})=H^{*}\left(\left(M \times E S^{1}\right) / S^{1}, \mathbb{Z}\right) .
$$

We only use cohomology with integer coefficients and write $H_{S^{1}}^{*}(M)=$ $H_{S^{1}}^{*}(M, \mathbb{Z})$.

If a circle action on $M$ is free then $H_{S^{1}}^{*}(M)=H^{*}\left(M / S^{1}\right)$.

The equivariant cohomology of a point with a trivial circle action is equal to the cohomology of the space $B S^{1}=E S^{1} / S^{1}$. (If $E S^{1}=S^{\infty}$, then $B S^{1}$ is the infinite dimensional projective space $\mathbb{C} P^{\infty}=S^{\infty} / S^{1}$.) It is known that $H_{S^{1}}^{*}(p t)=H^{*}\left(B S^{1}\right)$ is the ring of polynomials in one variable $\mathbb{Z}[u]$ where the generator $u$ has degree 2 .

The map $M \rightarrow p t$ produces the pullback map in equivariant cohomology $H_{S^{1}}^{*}(p t) \rightarrow H_{S^{1}}^{*}(M)$ which equips the equivariant cohomology ring with a $\mathbb{Z}[u]$-module structure.

A natural inclusion of $M$ into $\left(M \times E S^{1}\right) / S^{1}$ gives a map from the equivariant cohomology to the regular cohomology $\kappa: H_{S^{1}}^{*}(M) \rightarrow$ $H^{*}(M)$.

Given an invariant Morse function $f$ on $M$ and $a<b$, the exact sequence of the tuple $\left(M_{\leq a}, M_{\leq b}\right)$ is

$$
\ldots \rightarrow H_{S_{1}}^{*}\left(M_{\leq b}\right) \rightarrow H_{S_{1}}^{*}\left(M_{\leq a}\right) \stackrel{\delta}{\rightarrow} H_{S_{1}}^{*+1}\left(M_{\leq b}, M_{\leq a}\right) \rightarrow H_{S_{1}}^{*+1}\left(M_{\leq b}\right) \rightarrow \ldots
$$

To understand this exact sequence (especially the map $\delta$ ) better, let us describe the cohomology $H_{S_{1}}^{*}\left(M_{\leq b}, M_{\leq a}\right)$ in the case when $M_{(a, b)}$ contains a single critical point $p$ of index $\lambda$. By the classical results in invariant Morse theory (see [1]) we know that

$$
H_{S_{1}}^{*}\left(M_{\leq b}, M_{\leq a}\right)=H_{S^{1}}^{*}\left(W_{p, \geq a}^{-} W_{p, a}^{-}\right)=H_{S^{1}}^{*}\left(D^{\lambda}, S^{\lambda-1}\right),
$$

where $D^{\lambda}$ is the unit disc inside $\mathbb{C}^{\frac{\lambda}{2}}$ on which $S^{1}$ acts with weight $(1, \ldots, 1)$ and $S^{\lambda-1}=\partial D^{\lambda}$. By Thom isomorphism $H_{S^{1}}^{*}\left(D^{\lambda}, S^{\lambda-1}\right)=$ $H_{S^{1}}^{*-\lambda}(p t)$. Choosing a generator $\tau_{p}$ of $H_{S^{1}}^{\lambda}\left(D^{\lambda}, S^{\lambda-1}\right)$ we get a $\mathbb{Z}[u]$ module identification

$$
H_{S_{1}}^{*}\left(M_{\leq b}, M_{\leq a}\right)=\tau_{p} H_{S^{1}}^{*}(p t) .
$$

We call $\tau_{p}$ a Thom class of $p$. Notice that choosing $\tau_{p}$ is equivalent to picking an orientation on $W_{p}^{-}$. 
Similarly, if $M_{(a, b)}$ has a single critical circle $s$ of index $\lambda$ then

$$
H_{S^{1}}^{*}\left(M_{\leq b}, M_{\leq a}\right)=\tau_{s} H_{S^{1}}^{*}\left(S^{1}\right)=\tau_{s} H^{*}(p t)
$$

and $\tau_{s} \in H_{S^{1}}^{\lambda}\left(M_{\leq b}, M_{\leq a}\right)$ is a Thom class of $s$.

Lemma 5.1. Assume an action of $S^{1}$ on a compact oriented manifold $M$ is semi-free and $(f, \xi)$ is an invariant Morse-Smale function on $M$. Assume $a<b$ and $M_{(a, b)}$ contains a critical point $p$ of index $\lambda$ and $a$ critical circle $s$ of index $\lambda+1$. Let $f(p)<c<f(s)$. Suppose $M_{c}$ is oriented and $N=W_{p, c}^{+}, \nu(N)$ as well as $N^{\prime}=W_{s, c}^{-}, \nu\left(N^{\prime}\right)$ are equipped with compatible orientations.

Consider the boundary map of the exact sequence of the triple $\left(M_{\leq b}, M_{\leq c}, M_{\leq a}\right)$

$$
\delta: H_{S^{1}}^{*}\left(M_{\leq c}, M_{\leq a}\right) \rightarrow H_{S^{1}}^{*+1}\left(M_{\leq b}, M_{\leq c}\right) .
$$

This map takes $H_{S^{1}}^{\lambda}\left(M_{\leq c}, M_{\leq a}\right) \cong \tau_{p} \mathbb{Z}$ to $H_{S^{1}}^{\lambda+1}\left(M_{\leq b}, M_{\leq c}\right) \cong \tau_{s} \mathbb{Z}$. If $\delta\left(\tau_{p}\right)=c \tau_{s}$ then $N \cdot N^{\prime}= \pm c$.

Proof. Use excision to identify

$$
H_{S^{1}}^{*}\left(M_{\leq c}, M_{\leq a}\right)=H_{S^{1}}^{*}\left(M_{[a, c]}, M_{a}\right)
$$

and

$$
H_{S^{1}}^{*}\left(M_{\leq b}, M_{\leq c}\right)=H_{S^{1}}^{*}\left(M_{[c, b]}, M_{c}\right) .
$$

The map

$$
\kappa: H_{S^{1}}^{*}\left(M_{[a, c]}, M_{a}\right) \rightarrow H^{*}\left(M_{[a, c]}, M_{a}\right)
$$

is an isomorphism in degree $\lambda$. Moreover, by Poincare duality

$$
H^{\lambda}\left(M_{[a, c]}, M_{a}\right)=H_{\operatorname{dim} M-\lambda}\left(M_{[a, c]}, M_{c}\right) .
$$

Since $S^{1}$ acts freely on $M_{[c, b]}$ we have

$$
H_{S^{1}}^{*}\left(M_{[c, b]}, M_{c}\right)=H^{*}\left(M_{[c, b]} / S^{1}, M_{c} / S^{1}\right) .
$$

By Poincare duality

$$
H^{\lambda+1}\left(M_{[c, b]} / S^{1}, M_{c} / S^{1}\right) \cong H_{\operatorname{dim} M-\lambda-2}\left(M_{[c, b]} / S^{1}, M_{b} / S^{1}\right) .
$$

Thus, we view $\delta$ as the map

$$
\delta: H_{\operatorname{dim} M-\lambda}\left(M_{[a, c]}, M_{c}\right) \rightarrow H_{\operatorname{dim} M-\lambda-2}\left(M_{[c, b]} / S^{1}, M_{b} / S^{1}\right) .
$$

This map has a geometric interpretation. The generator of $H_{\operatorname{dim} M-\lambda}$ $\left(M_{[a, c]}, M_{c}\right)$ is given by the cycle $W_{p,[a, c]}^{+}$, then $\delta$ sends it to the cycle $\left(\partial W_{p,[a, c]}^{+}\right) / S^{1}=W_{p, c}^{+} / S^{1}=N / S^{1}$ inside the tuple $\left(M_{[c, b]} / S^{1}, M_{b} / S^{1}\right)$. 
Apply [8, Lemma 7.2] to our situation. It states that the homology class of $N / S^{1}$ is a generator of $H_{\operatorname{dim} M-\lambda-2}\left(M_{[c, b]} / S^{1}, M_{b} / S^{1}\right)$ multiplied by $N \cdot N^{\prime}$ which proves the lemma.

Theorem 5.2. Assume an action of $S^{1}$ on a compact oriented manifold $M$ is semi-free and $(f, \xi)$ is an invariant Morse-Smale function. For $a<b$ suppose $M_{[a, b]}$ is connected. Assume the set of critical points in $M_{(a, b)}$ consists of critical points $p_{1}, \ldots, p_{k}$ of the same index $\lambda$ with $2 \leq \lambda \leq \operatorname{dim} M-2$ (or critical circles $s_{1}, \ldots, s_{k}$ of the same index $\lambda$ with $3 \leq \lambda \leq \operatorname{dim} M-2)$. Given a basis $\tau_{1}, \ldots, \tau_{k}$ of $H_{S_{1}}^{\lambda}\left(M_{\leq b}, M_{\leq a}\right)$ there exists an invariant Morse-Smale function $\left(f^{\prime}, \xi^{\prime}\right)$ which coincides with $(f, \xi)$ outside $M_{[a, b]}$, has the same set of critical points as $(f, \xi)$ and after choosing the proper orientations on $W_{p_{i}}^{-}$the Thom class of each $p_{i}$ is $\tau_{i}$ (or the Thom class of each $s_{i}$ is $\tau_{i}$ ).

Proof. Consider the case when $M_{(a, b)}$ contains only critical points $p_{1}, \ldots, p_{k}$. Forget for a moment about the circle acting on our manifold and try to prove the theorem for the regular cohomology groups. Then using excision and Poincare duality we can identify

$$
H^{\lambda}\left(M_{\leq b}, M_{\leq a}\right)=H_{n-\lambda}\left(M_{[a, b]}, M_{b}\right) .
$$

Moreover, the Thom classes of the fixed point $p_{i}$ are given by the cycles $W_{p_{i},[a, b]}^{+}$. Translating our theorem into a statement about a basis in homology leads to a statement identical to [8, Theorem 7.6]. In particular, there exists a Morse-Smale function $(\tilde{f}, \tilde{\xi})$ on $M$ which coincides with $(f, \xi)$ outside $M_{[a, b]}$ and the Thom classes (in regular cohomology) of $p_{i}$ 's are exactly $\kappa\left(\tau_{i}\right)$ 's

Notice that the map $\kappa$ identifies $H_{S^{1}}^{\lambda}\left(M_{\leq b}, M_{\leq a}\right)$ with $H^{\lambda}\left(M_{\leq b}, M_{\leq a}\right)$. So, if we show $\left(f^{\prime}, \xi^{\prime}\right)$ can be chosen invariantly with respect to the circle action, the theorem is proved.

Without loss of generality we may assume $(\tilde{f}, \tilde{\xi})$ coincides with $(f, \xi)$ in a neighborhood of every fixed point, this follows from an examination of the proof of [8, Theorem 7.6].

Let us average $(\tilde{f}, \tilde{\xi})$ with respect to the circle action to produce invariant function $f^{\prime}$ and invariant vector field $\xi^{\prime}$. Then $\left(f^{\prime}, \xi^{\prime}\right)$ is identical to $(f, \xi)$ outside of $M_{[a, b]}$ and in neighborhoods of the fixed points $\left\{p_{1}, \ldots, p_{k}\right\}$. Moreover, it is obvious that $\xi^{\prime}\left(f^{\prime}\right)<0$ on $M-M^{S^{1}}$. Hence $f^{\prime}$ is an invariant Morse function and $\xi^{\prime}$ is its invariant gradientlike vector field. Moreover $\kappa\left(\tau_{p_{i}}\right)$ are the same as for $(\tilde{f}, \tilde{\xi})$. Hence the Thom classes $\tau_{p_{i}}$ form the required basis. 
Finally, to make $\left(f^{\prime}, \xi^{\prime}\right)$ into an invariant Morse-Smale function we need to apply Theorem 2.1 and perturb $\xi^{\prime}$ without changing the Thom classes $\tau_{p_{i}}$.

Let us now outline the proof in the case when $M_{(a, b)}$ contains only critical circles. Since the circle acts freely on $M_{[a, b]}$, we can make an analogous statement on $M_{[a, b]} / S^{1}$ and then use Poincare duality to translate it into a statement in homology. This statement will follow from [8, Theorem 7.6], which is proved by constructing a series of isotopies of identity on $M_{a}$ and then changing the vector field according to these isotopies. Inspection of the proof of [8, Theorem 7.6] shows that each of these isotopies is identity outside a contractible set and hence can be lifted to $M_{[a, b]}$. This adopts the proof of [8, Theorem 7.6] to give a proof of our theorem in the case of critical circles.

\section{Proof of the main theorem.}

Lemma 6.1. Assume a circle action on a compact manifold $M$ is torsion-free and has isolated fixed points $p_{1}, \cdots, p_{m}$. Choose invariant neighborhoods $U_{i}$ of $p_{i}$ diffeomorphic to unit balls and set $U=\cup_{i} U_{i}$. Then the homology groups $H_{*}\left((M-U) / S^{1}, U / S^{1}\right)$ are $\mathbb{Z}$-torsion-free.

Proof. By Poincare duality

$$
H_{*}\left((M-U) / S^{1}, \partial U / S^{1}\right)=H^{\operatorname{dim} M-1-*}\left((M-U) / S^{1}\right) .
$$

Since

$$
H^{*}\left((M-U) / S^{1}\right)=H_{S^{1}}^{*}(M-U)=H_{S^{1}}^{*}\left(M-M^{S^{1}}\right),
$$

it is enough to show that cohomology groups $H_{S^{1}}^{*}\left(M-M^{S^{1}}\right)$ are torsionfree.

Consider the Mayer-Vietoris sequence for $M=U \cup\left(M-M^{S^{1}}\right)$ :

$$
\ldots \rightarrow H_{S^{1}}^{*}(M) \rightarrow H_{S^{1}}^{*}(U) \oplus H_{S^{1}}^{*}\left(M-M^{S^{1}}\right) \rightarrow H_{S^{1}}^{*}\left(U \cap\left(M-M^{S^{1}}\right)\right) \rightarrow \ldots
$$

An easy computation in equivariant cohomology implies the surjectivity of the map

$$
\oplus_{i} H_{S^{1}}^{*}\left(p_{i}\right) \cong H_{S^{1}}^{*}(U) \rightarrow H_{S^{1}}^{*}\left(U \cap\left(M-M^{S^{1}}\right)\right) \cong H^{*}\left(\partial U / S^{1}\right) .
$$

Hence the Mayer-Vietoris sequence breaks up into short exact sequences. Since $H_{S^{1}}^{*}(M)$ and $H_{S^{1}}^{*}\left(U \cap\left(M-M^{S^{1}}\right)\right)$ are torsion-free, the same holds for $H_{S^{1}}^{*}\left(M-M^{S^{1}}\right)$. 
An invariant Morse function $f$ is self-indexing if $f(q)=\sigma(q)$ for every $q \in \operatorname{Crit}_{c}(f)$, in particular, the critical values of $f$ are between zero and $\operatorname{dim} M$. For an integer $k$, define $M_{k}=M_{\leq k+\frac{1}{2}}$. Then $H_{S^{1}}^{*}\left(M_{k}, M_{k-1}\right)$ is generated as a $\mathbb{Z}[u]$ module by the Thom classes of critical points and circles of index $k$. Then, by definition, $f$ is perfect on $M_{k}$ if $\mathcal{P}_{S^{1}}\left(M_{k}\right)=\mathcal{M}_{S^{1}}\left(M_{k}, f\right)$. The exact sequence of the triple $\left(M_{k+1}, M_{k}, M_{k-1}\right)$ defines the map

$$
\delta_{k}: H_{S^{1}}^{*}\left(M_{k-1}, M_{k-2}\right) \rightarrow H_{S^{1}}^{*+1}\left(M_{k}, M_{k-1}\right)
$$

Proof of Theorem 1.1. The proof will use induction on $k$ to construct a self-indexing invariant Morse-Smale function $(f, \xi)$ on $M$, such that

1) $f$ is perfect on $M_{k}$,

2) $\delta_{m}\left(\tau_{s}\right)=0$ for every critical circle $s$ of index $m-1$.

Basis of induction for $k=0$. Use the notations from Lemma 6.1. Since $M$ is simply-connected, it is easy to see using van Kampen theorem that $(M-U) / S^{1}$ is simply-connected as well. This together with Lemma 6.1 allow to use $[\mathbf{9}$, Theorem 6.5$]$ which implies that there exists a perfect Morse function

$$
\tilde{f}:\left((M-U) / S^{1},(\partial U) / S^{1}\right) \rightarrow([1, \infty), 1) .
$$

Lift $\tilde{f}$ to $f$ on $M-U$. As in the proof of Theorem 3.1 extend $f$ to $M$ by setting $f=\phi_{i}^{*}\left(|z|^{2}\right)$ on $U_{i}$ and smoothing it along $\partial U$.

By Theorem 3.4 there exists an invariant gradient-like vector field $\xi$, such that $(f, \xi)$ is an invariant Morse-Smale function. By Theorem 3.2 we can assume $f$ is self-indexing. Conditions (1) and (2) are satisfied by $f$, since $\tilde{f}$ is a perfect Morse function.

Induction step for $k=1$. Assume $f$ satisfies conditions (1) and (2) for $k=0$. Let $p$ be a fixed point of index 0 such that $\delta_{1}\left(\tau_{p}\right) \neq 0$. Then it is easy to see that there exists a critical circle $s$ of index 1 such that the closure of the intersection $W_{s}^{-} \cap W_{p}^{+}$is a single disc. By Theorem 4.2 we can cancel the critical circle $s$ by increasing the index of $p$ by 2 . We can continue this process until the map $\delta_{1}$ is nontrivial. After this $(f, \xi)$ will satisfy (1) and (2) for $k=1$.

Induction step for even $k \geq 2$. If $k$ is even and $(f, \xi)$ satisfies conditions (1) and (2) for $k-1$, then $(f, \xi)$ satisfies (1) and (2) for $k$ as well. Indeed, the second condition does not depend on $k$. At the same time, the map $\delta_{k}$ is trivial, by condition (2) and the fact that $M_{k-1}-M_{k-2}$ contains no fixed points. This implies that the 
exact sequence of the pair $\left(M_{k}, M_{k-1}\right)$ splits into short exact sequences proving the perfectness of $f$ on $M_{k}$.

Induction step for odd $k \geq 1$. Assume $(f, \xi)$ satisfies (1) and (2) for $k-1$. Since the map $\delta_{k}$ vanishes on all the Thom classes of critical circles we can assume without loss of generality that there are no critical circles of index $k-1$. Assume $p_{i_{1}}, \ldots, p_{i_{\ell}}$ are all the fixed point of index $k-1$ of $f$.

Choose a basis $\tau_{1}, \ldots, \tau_{q}$ of the kernel of the map $\delta_{k}$ restricted to $H^{k-1}\left(M_{k-1}, M_{k-2}\right)$. Complete it to a basis of $H^{k-1}\left(M_{k-1}, M_{k-2}\right)$ by adding $\tau_{q+1}, \ldots, \tau_{\ell}$. By Theorem 5.2 we can perturb $(f, \xi)$ on $M_{k-1}-$ $M_{k-2}$ such that $\tau_{p_{i}}=\tau_{i}$ after the perturbation.

Assume $s_{1}, \ldots, s_{r}$ are the critical circles of $f$ of index $k$, then their Thom classes form a basis of $H^{k}\left(M_{k}, M_{k-1}\right)$. The elements $\tilde{\tau}_{j}=\delta_{k}\left(\tau_{q+j}\right)$ of $H^{k}\left(M_{k}, M_{k-1}\right)$ span the image of $\delta_{k}$. Complete $\tilde{\tau}_{1}, \ldots \tilde{\tau}_{\ell-q}$ to a basis of $H^{k}\left(M_{k}, M_{k-1}\right)$ by adding $\tilde{\tau}_{\ell-q+1}, \ldots, \tilde{\tau}_{r}$. By Theorem 5.2 we can alter $(f, \xi)$ on $M_{k}-M_{k-1}$ to guarantee $\tau_{s_{i}}=\tau_{i}$. Apply Lemma 5.1 together with Theorem 2.2 to cancel the critical circles $s_{1}, \ldots s_{\ell-q}$ by increasing the index of each $p_{q+i}$ by 2 . This produces a new invariant Morse-Smale function which satisfies (1) and (2) for $k$.

The above argument works as long as the assumptions of Theorems 2.2 are satisfied. Checking these assumptions boils down to making sure that if $f$ is perfect on $M_{k}$ for $k>1$, then $\left(f^{-1}(a)\right) / S^{1}$ for any regular $a<k+\frac{1}{2}$ is simply-connected. It is enough to prove that if the interval $[a, b]$ contains exactly one critical value then $\pi_{1}\left(f^{-1}(a) / S^{1}\right)=$ $\pi_{1}\left(f^{-1}(b) / S^{1}\right)$. This follows from Theorem 3.2, Lemma 4.5 and an analogue of Lemma 4.5 for critical circle instead of fixed point with an almost identical proof.

Remark 6.2. The dimension restriction, which appears in this theorem comes from the dimension restrictions in classical Morse theory. In particular, if $\operatorname{dim} M$ is either 4 or 6 then [9, Theorem 6.5] used in the basis of induction fails. 


\section{Bibliography}

[1] Atiyah, M., Bott R. The Yang-Mills equations over Riemann surfaces. Philos. Trans. Roy. Soc. London Ser. A 308 (1983), no. $1505,523-615$.

[2] M. F. Atiyah, R. Bott. The moment map and equivariant cohomology Topology 23 (1984), no. 1, 1-28.

[3] Burghelea, D. A short course on Witten Helffer-Sjöstrand theory. arXiv:math.DG/0101063

[4] Hattori, A. Symplectic manifolds with semi-free Hamiltonian $S^{1}$ action. Tokyo J. Math. 15 (1992), no. 2, 281-296.

[5] L. Jeffrey, F. Kirwan. Localization for nonabelian group actions. Topology Vol. 34, No 2, pp. 291-327, 1995

[6] Kirwan, F. Cohomology of quotients in symplectic and algebraic geometry. Mathematical Notes, 31. Princeton University Press, Princeton, NJ, 1984

[7] Kogan, M. Localization for invariant submanifolds. Math. Res. Lett. 8 (2001), no. 1-2, 141-156

[8] Milnor, J. Lectures on the h-cobordism theorem. Notes by L. Siebenmann and J. Sondow Princeton University Press, Princeton, N.J. 1965

[9] Smale, S. On the structure of manifolds. Amer. J. Math. 841962 387-399.

[10] Tolman S., Weitsman J. On semifree symplectic circle actions with isolated fixed points. Topology 39 (2000), no. 2, 299-309.

Department of Mathematics, Northeastern University, Boston, MA 02115

E-mail address: misha@neu.edu

The author is supported by an NSF Postdoctoral Fellowship. 